\title{
Prediction of the Number of Women Population in Medan City 2025 by Using the Leslie Matrix
}

\author{
Mulyono*, Abil Mansyur, Faridawaty Marpaung \\ Mathematics Study Program, Faculty of Mathematics and Natural Sciences, Medan State University, Indonesia
}

Received October 26, 2020; Revised January 18, 2021; Accepted January 28, 2021

\section{Cite This Paper in the following Citation Styles}

(a): [1] Mulyono, Abil Mansyur, Faridawaty Marpaung, "Prediction of the Number of Women Population in Medan City 2025 by Using the Leslie Matrix," Universal Journal of Applied Mathematics, Vol. 9, No. 1, pp. 1 - 9, 2021. DOI: 10.13189/ujam.2021.090101.

(b): Mulyono, Abil Mansyur, Faridawaty Marpaung (2021). Prediction of the Number of Women Population in Medan City 2025 by Using the Leslie Matrix. Universal Journal of Applied Mathematics, 9(1), 1 - 9. DOI: 10.13189/ujam.2021.090101.

Copyright $C 2021$ by authors, all rights reserved. Authors agree that this article remains permanently open access under the terms of the Creative Commons Attribution License 4.0 International License

\begin{abstract}
Population problems can cause problems, both in terms of political, economic, socio-cultural life, defense and security, as well as other aspects of life concerning the use of natural resources and the environment. By knowing the population growth rate, the Medan city government can implement a policy to anticipate social problems that may arise. This study aims to determine the number and rate of female population growth in the city of Medan in 2025. The Leslie matrix model is a model that can be used to predict the number and rate of female population growth. The step taken to predict the number of population $p$ in the following year is to form a column vector whose entry is the initial number of population for each age class. Next look for $n(t+p)$ which is the total population for the following year using the formula $n(t+p)=A^{p} n(t)$ where $A$ is the Leslie matrix. Furthermore, to predict the population growth rate using the Leslie matrix is to find the positive eigenvalues $\lambda$ of the matrix $A$. Based on positive eigenvalues $\lambda$, three cases occur, namely: 1 ) the population will tend to increase if $\lambda>1$;) the population will tend to decline if $\lambda<1 ; 3$ ) the population will tend to be stable if $\lambda=1$. Based on data analysis, the total female population in the city of Medan in 2025 is 1,576,294. Furthermore, the eigenvalues of $\lambda=1,3673$ which mean that the number of female populations in the city of Medan tends to increase.
\end{abstract}

Keywords Leslie Matrix, Population Growth, Eigenvalues, Eigenvectors

\section{Introduction}

Medan City is the capital of North Sumatra Province. This city is the third-largest city in Indonesia after Jakarta and Surabaya, as well as the largest city outside Java Island with an area of $265.10 \mathrm{~km}^{2}$. Based on data from the Central Statistics Agency (BPS) for Medan City in 2018 there is 2.264.145 soul, which consists of 1.118 .402 soul man and1.145.743 female soul. Compared to the total population in 2017 , there was a population increase of16.720 souls $(0,74$ percent $)$ and the population density reached 8,541 people $/ \mathrm{km}^{2}$. With the population density figure of the city of Medan, it may cause a lot of problems.

The population is an important asset of an area, but it should be noted that uncontrolled population growth will become a problem. The population problem in Indonesia has reached a disturbing level, both in terms of political, economic, socio-cultural life, defense and security, as well as other aspects of life about the use of natural resources and the environment, for example, uneven population density, large numbers of people. the number of unemployed and high population growth. Population problems in a narrow sense are always related to the number, structure, composition, and dynamic processes of the local population

With the problems described above, the government, especially the Medan City government, needs to be prepared and meet the needs of its citizens as a form of responsible government. Of course, the amount of effort the government has made is based on data and 
information, one of which is the rate of population and population growth in recent years. By knowing the population growth rate of Medan City in the past years, the city government can anticipate it in the long term by predicting the population for the next few years. Population predictions and population forecasts are often used as interchangeable terms. However, these two terms have very basic differences. Various literature states population projections as predictions (forecasts) which are based on certain rational assumptions built for future trends using statistical tools or mathematical calculations. On the other hand, population forecasting can be with or without assumptions or calculations. Without certain conditions or conditions or certain approaches.

By knowing the prediction of the number and rate of population growth, whether population growth is increasing, decreasing or remaining stable in the coming years, it will affect the development carried out which aims to provide clothing and food needs as basic needs, various educational, health and various other social facilities sufficient and evenly distributed in the context of increasing welfare. The population growth rate is the change in the number of people in a certain area each year.

Many models can be used to explain population growth. One of the models used by population experts is the Leslie model. Where this model uses a mathematical approach, namely the matrix. The Leslie matrix model is one of the models used by demographers, which was discovered by an ecologist named PH Leslie in 1945. The Leslie matrix has a unique shape, namely the Leslie matrix in the form of a square matrix with the first-row entry of the Leslie matrix consisting of fertility levels. female, the sub-diagonal contains the female's survival rate and the other entries are zero.

The Leslie Matrix Model can provide an overview of the dynamics of the process of growing a population in the long run, among others: regarding population growth, in the long run, the distribution of the population in age groups for the long term, and its application to a harvesting policy for a growing population [4]. The Leslie matrix is only used for one sex, and it is usually women who are considered. Because women reproduce, that means birth and birth will cause an increase in population

There are several studies on the Leslie matrix, among others, Yudha Pratama [7] conducted a study in East Java and the results showed several factors that influence population growth, namely fertility, survival, and age vulnerability of the population. Furthermore, Wahidah Sanusi, Sukarna and Nur Ridiawati [8] used the Leslie matrix to predict the number and rate of population growth in the city of Makassar in 2018. Their results showed that the number of female populations in the city of Makassar has always increased. Based on the description above and to avoid population problems, the Medan city government needs to know the prediction of the number and growth rate of Medan's population for the next few years.

\section{Leslie's Matrix}

One of the most common models of population growth used by population experts is the Leslie model. This model describes the growth of a human or animal population. The Leslie Matrix was discovered by an ecologist named P. H Leslie in 1945. The Leslie matrix can provide an overview of the dynamics of a population growth process, including population growth in the long run, distribution of population in age groups for the long term, and its application. on a harvesting policy for an established population.

Leslie's model using the following assumptions:

1. Only consider the number of female / female population.

2. The maximum age that can be reached by each individual is $n$ year.

3. Age group of the population.

4. Endurance life (Survival Rate) for each age group to the next stage of age known.

5. Birth rate for each age group is known.

6. The distribution of initial ages is known

Define the age distribution $x^{k}$ at time with $t_{k}$

$$
x^{(k)}=\left[\begin{array}{c}
x_{1}^{(k)} \\
x_{2}^{(k)} \\
x_{3}^{(k)} \\
\vdots \\
x_{n}^{(k)}
\end{array}\right]
$$

where $x_{i}^{(k)}$ is the number of girls in the age class at time. Thus, at the time, the number of girls in the first age class, only girls born between the time of and. The number of offspring produced for each age class can be calculated by multiplying the reproductive number for the age class by the number of girls in that age class for each group. The sum of these values gives the total number of offspring produced. Thus, it can be written mathematically as

$$
x_{1}^{(k)}=L_{1} x_{1}^{(k-1)}+L_{2} x_{2}^{(k-1)}+\cdots+L_{n} x_{n}^{(k-1)}
$$

which states that the number of girls of age class 1 is equal to the number of girls born to girls aged class 1 between time and plus the number of girls born to girls aged class 2 between time and onwards plus the number of girls born to girls in age class - $\mathrm{n}$ between time $t_{k}$ and $t_{k-1}$.

The number of women in the second age class group at the time of $t_{k}$ were women in the first age class group at the time of $t_{k-1}$ who were still alive at the time of $t_{k}$, or mathematically written as, $x_{2}^{(k)}=P_{1} x_{1}^{(k-1)}$. Furthermore, the number of women in the 3rd age class group at the time of $t_{k}$ were women in the first age class group at that time of $t_{k-1}$ who were still alive at the time of $t_{k}$, or mathematically written as, $x_{3}^{(k)}=P_{2} x_{2}^{(k-1)}$, 
in general, the number of women in the nth age class group at the time of $t_{k}$ was women in the age class group $(n-1)$ at that time $t_{k-1}$ who were still alive at that time of $t_{k}$, or mathematically written with, $x_{n}^{(k)}=P_{n-1} x_{n-1}^{(k-1)}$. The description above can be expressed in the form of a linear equation system, namely;

$$
\begin{gathered}
x_{1}^{(k)}=L_{1} x_{1}^{(k-1)}+L_{2} x_{2}^{(k-1)}+\cdots+L_{n} x_{n}^{(k-1)} \\
x_{2}^{(k)}=P_{1} x_{1}^{(k-1)} \\
x_{3}^{(k)}=P_{2} x_{2}^{(k-1)} \\
x_{n}^{(k)}=P_{n-1} x_{n-1}^{(k-1)}
\end{gathered}
$$

or in the form of a matrix to be

$$
\left[\begin{array}{c}
x_{1}^{(k)} \\
x_{2}^{(k)} \\
x_{3}^{(k)} \\
\cdot \\
\cdot \\
x_{n}^{(k)}
\end{array}\right]=\left[\begin{array}{ccccc}
a_{1} & a_{2} & \ldots & a_{n-1} & a_{n} \\
b_{1} & 0 & \ldots & 0 & 0 \\
0 & b_{2} & \ldots & 0 & 0 \\
\cdot & \cdot & \ldots & \cdot & \cdot \\
\cdot & \cdot & \ldots & \cdot & \cdot \\
\cdot & \cdot & \ldots & \cdot & \cdot \\
0 & 0 & \ldots & b_{n-1} & 0
\end{array}\right]\left[\begin{array}{c}
x_{1}^{(k-1)} \\
x_{2}^{(k-1)} \\
x_{3}^{(k-1)} \\
\cdot \\
\cdot \\
x_{n}^{(k-1)}
\end{array}\right]
$$

or shorter is written as

$$
x^{(k)}=L x^{(k-1)}
$$

Where

$$
x^{(k)}=\left[\begin{array}{c}
x_{1}^{(k-1)} \\
x_{2}^{(k-1)} \\
x_{3}^{(k-1)} \\
\vdots \\
x_{n}^{(k-1)}
\end{array}\right] \text {, is the vector of the age distribution at time }
$$$$
t_{k}
$$$$
x^{(k-1)}=\left[\begin{array}{c}
x_{1}^{(k-1)} \\
x_{2}^{(k-1)} \\
x_{3}^{(k-1)} \\
\vdots \\
x_{n}^{(k-1)}
\end{array}\right] \text {, is the vector of the age distribution at }
$$$$
\text { time } t_{k-1}
$$

and

$$
L=\left[\begin{array}{cccccc}
a_{1} & a_{2} & a_{3} & \cdots & a_{n-1} & a_{n} \\
b_{1} & 0 & 0 & \cdots & 0 & 0 \\
0 & b_{2} & 0 & \cdots & 0 & 0 \\
\vdots & \vdots & \vdots & & \vdots & \vdots \\
0 & 0 & 0 & \cdots & b_{n-1} & 0
\end{array}\right]
$$

called the Leslie matrix.

The birth process and the death process between two consecutive observations can be explained using the demographic parameters as shown in Table 1.
Table 1. Parameters in the Leslie Matrix

\begin{tabular}{|c|c|}
\hline Model Parameters & Information \\
\hline$a_{i}, i=1,2, \cdots, n$ & $\begin{array}{c}\text { Average number of girls born to a } \\
\text { woman during her age group }-i\end{array}$ \\
\hline$b_{i}, i=1,2, \cdots, n$ & $\begin{array}{c}\text { The number of women in the it age } \\
\text { group that can be expected to be alive } \\
\text { and up to the it age group }\end{array}$ \\
\hline
\end{tabular}

Based on the definition, it will be found that (i) $a_{i} \geq 0$ for $i=1,2, \cdots, n$ and (ii) $0<b_{i} \leq 1$ for $i=1,2, \cdots, n$. It can be seen that it is not permissible to allow the existence of being equal to zero, because if this were to occur there would be no woman or female alive after age group i. It is also assumed that at least one is positive so that birth will occur. Each age group for which the value is positive is called the reproductive age group.

To determine the prediction of population growth rate, further investigation of the eigenvalues of the Leslie matrix can be done. The eigenvalues of the Leslie matrix can indicate that the population growth rate tends to increase, decrease, or remain.

Theorem 2.1 The Leslie matrix has a single positive eigenvalue $\lambda_{1}$, this eigenvalue has multiplicity and is an eigenvector $\boldsymbol{x}_{\mathbf{1}}$ all of the entries are positive.

\section{Proof :}

The eigenvalues of the Leslie matrix are the roots of the characteristic polynomial equation of the Leslie matrix. The characteristic equation of the matrix can be written as follows:

$$
\begin{aligned}
& p(\lambda)=|\lambda I-L|=0 \\
& \lambda\left[\begin{array}{ccccc}
1 & 0 & 0 & \cdots & 0 \\
0 & 1 & 0 & \cdots & 0 \\
0 & 0 & 1 & \cdots & 0 \\
\vdots & \vdots & \vdots & \ddots & \vdots \\
0 & 0 & 0 & \cdots & 1
\end{array}\right]-\left[\begin{array}{ccccc}
a_{1} & a_{2} & \cdots & a_{i-1} & a_{i} \\
b_{1} & 0 & \cdots & 0 & 0 \\
0 & b_{2} & \cdots & 0 & 0 \\
\vdots & \vdots & \ddots & \vdots & \vdots \\
0 & 0 & 0 & b_{i-1} & 0
\end{array}\right] \mid=0 \\
& \left|\left[\begin{array}{ccccc}
\lambda & 0 & 0 & \cdots & 0 \\
0 & \lambda & 0 & \cdots & 0 \\
0 & 0 & \lambda & \cdots & 0 \\
\vdots & \vdots & \vdots & \ddots & \vdots \\
0 & 0 & 0 & \cdots & \lambda
\end{array}\right]-\left[\begin{array}{ccccc}
a_{1} & a_{2} & \cdots & a_{i-1} & a_{i} \\
b_{1} & 0 & \cdots & 0 & 0 \\
0 & b_{2} & \cdots & 0 & 0 \\
\vdots & \vdots & \ddots & \vdots & \vdots \\
0 & 0 & 0 & b_{i-1} & 0
\end{array}\right]\right|=0 \\
& \lambda^{n}-a_{1} \lambda^{n-1}-a_{2} b_{1} \lambda^{n-2}-\cdots \\
& -a_{i} b_{1} b_{2} \cdots b_{i-1}=0
\end{aligned}
$$

Equation (5) divided by $\lambda^{n}$, then we get:

$$
\Leftrightarrow 1-\frac{a_{1}}{\lambda}-\frac{a_{2} b_{1}}{\lambda^{2}}-\frac{a_{3} b_{1} b_{2}}{\lambda^{3}}-\cdots-\frac{a_{i} b_{1} b_{2} \cdots b_{i-1}}{\lambda^{n}}=0
$$




$$
\Leftrightarrow \frac{a_{1}}{\lambda}+\frac{a_{2} b_{1}}{\lambda^{2}}+\frac{a_{3} b_{1} b_{2}}{\lambda^{3}}+\cdots+\frac{a_{i} b_{1} b_{2} \cdots b_{i-1}}{\lambda^{n}}=1
$$

Consider a polynomial equation

$$
\begin{array}{r}
h(\lambda)= \\
a_{1} \lambda^{-1}+a_{2} b_{1} \lambda^{2}+a_{3} b_{1} b_{2} \lambda^{3}+\cdots+a_{i} b_{1} b_{2} \cdots b_{i-1} \lambda^{-n}=
\end{array}
$$

So that it is obtained

$$
h(\lambda)=1
$$

It is known $a_{i}, b_{i}$ to be positive. If the eigenvalues of the Leslie matrix $\lambda_{i}$ are substituted for equation (6), let $\lambda_{i}$ be positive for $0 \leq i \leq \infty$, then the values of $h(\lambda)$ will go towards zero and the monotone go down

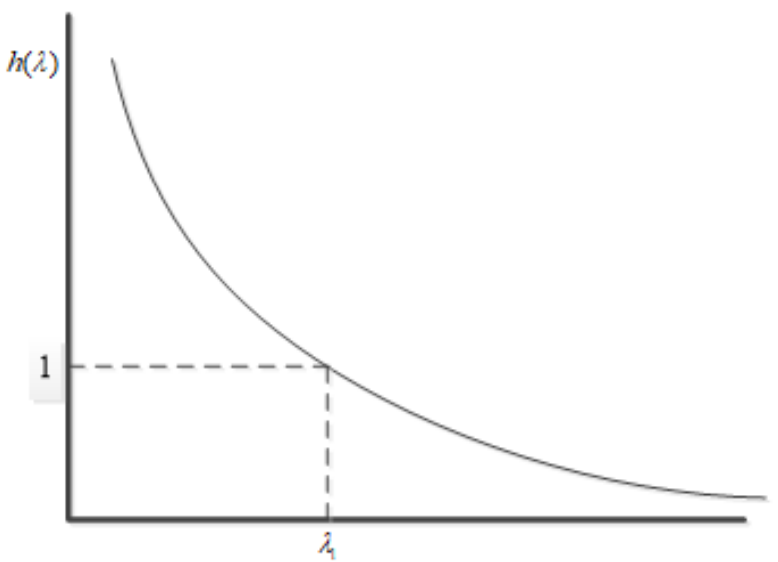

Figure 1. Graph of the $h(\lambda)$ function

From Figure 1, it is obtained that the value of each eigenvalue has exactly one solution value at $h\left(\lambda_{1}\right)$. So it can be concluded that the eigenvalues of the Leslie matrix differ from one another and there is a positive $\lambda$ that is single, for example $\lambda=\lambda_{1}$ and $q\left(\lambda_{1}\right)=1$. Obtained also $\lambda_{1}$ has multiplicity equal to one. $h\left(\lambda_{1}\right)$

Given $\boldsymbol{x}_{\mathbf{1}}$ is an eigenvector of A corresponding to that which satisfies $\lambda_{1}$

$$
\left(\lambda_{1} 1-A\right) x_{1}=0 .
$$

For example

$$
\boldsymbol{x}_{\mathbf{1}}=\left[\begin{array}{c}
x_{1} \\
x_{2} \\
x_{3} \\
\vdots \\
x_{n}
\end{array}\right]
$$

So that

$$
\begin{aligned}
& \left(\left[\begin{array}{ccccc}
\lambda_{1} & 0 & 0 & \cdots & 0 \\
0 & \lambda_{1} & 0 & \cdots & 0 \\
0 & 0 & \lambda_{1} & \cdots & 0 \\
\vdots & \vdots & \vdots & \ddots & \vdots \\
0 & 0 & 0 & \cdots & \lambda_{1}
\end{array}\right]\right. \\
& \left.-\left[\begin{array}{cccrc}
a_{1} & a_{2} & \cdots & a_{i-1} & a_{i} \\
b_{1} & 0 & \cdots & 0 & 0 \\
0 & b_{2} & \cdots & 0 & 0 \\
\vdots & \vdots & \ddots & & \vdots \\
0 & 0 & 0 & b_{i-1} & 0
\end{array}\right]\right) \boldsymbol{x}_{\mathbf{1}}=\mathbf{0}
\end{aligned}
$$$$
\left[\begin{array}{ccclc}
\lambda_{1}-a_{1} & -a_{2} & \cdots & -a_{i-1} & -a_{i} \\
-b_{1} & \lambda_{1} & \cdots & 0 & 0 \\
0 & -b_{2} & \ddots & 0 & 0 \\
\vdots & \vdots & \ddots & \ddots & \vdots \\
0 & 0 & 0 & -b_{i-1} & \lambda_{1}
\end{array}\right] \boldsymbol{x}_{\mathbf{1}}=\mathbf{0}
$$$$
\left[\begin{array}{ccclc}
\lambda_{1}-a_{1} & -a_{2} & \cdots & -a_{i-1} & -a_{i} \\
-b_{1} & \lambda_{1} & \cdots & 0 & 0 \\
0 & -b_{2} & \ddots & 0 & 0 \\
\vdots & \vdots & \ddots & \ddots & \vdots \\
0 & 0 & 0 & -b_{i-1} & \lambda_{1}
\end{array}\right]\left[\begin{array}{c}
x_{1} \\
x_{2} \\
x_{3} \\
\vdots \\
x_{n}
\end{array}\right]=\mathbf{0}
$$

$$
\left[\begin{array}{c}
\left(\lambda_{1}-a_{1}\right) x_{1}-a_{2} x_{2}-\cdots-a_{i-1} x_{n-1}-a_{i} x_{n} \\
-b_{1} x_{1}+\lambda_{1} x_{2} \\
-b_{2} x_{2}+\lambda_{1} x_{3} \\
\vdots \\
-b_{i-1} x_{n-1}+\lambda_{1} x_{n}
\end{array}\right]=\left[\begin{array}{c}
0 \\
0 \\
\vdots \\
0 \\
0
\end{array}\right]
$$

then obtained

$$
\begin{gathered}
-b_{1} x_{1}+\lambda_{1} x_{2}=0 \Leftrightarrow x_{2}=\frac{b_{1} x_{1}}{\lambda_{1}} \\
\Leftrightarrow x_{2}=\frac{b_{1} x_{1}}{\lambda_{1}} \\
-b_{2} x_{2}+\lambda_{1} x_{3}=0 \\
\Leftrightarrow x_{3}=\frac{b_{2} x_{2}}{\lambda_{1}} \Leftrightarrow x_{3}=\frac{b_{1} b_{2} x_{1}}{\lambda_{1}{ }^{2}} \\
-b_{i-2} x_{n-2}+\lambda_{1} x_{n-1}=0 \\
\Leftrightarrow x_{n-1}=\frac{b_{i-2} x_{n-2}}{\lambda_{1}} \\
\Leftrightarrow x_{n-1}=\frac{b_{1} b_{2} x_{1} \ldots b_{1-2} x_{1}}{\lambda_{1}{ }^{n-2}} \\
-b_{i-1} x_{n-1}+\lambda_{1} x_{n}=0 \\
\Leftrightarrow x_{n}=\frac{b_{i-1} x_{n-1}}{\lambda_{1}} \Leftrightarrow x_{n}=\frac{b_{1} b_{2} \cdots b_{i-1} x_{1}}{\lambda_{1}{ }^{n-1}}
\end{gathered}
$$

If equation (8), (9), (10), (11) is substituted for equation (7), then we get:

$$
\begin{aligned}
& \lambda_{1} x_{1}-a_{1} x_{1}-a_{2}=\frac{b_{1} x_{1}}{\lambda_{1}}-a_{3} \\
= & \frac{b_{1} b_{2} x_{1}}{\lambda_{1}{ }^{2}}-\cdots-a_{n-1} \frac{b_{1} b_{2} \cdots b_{1-2} x_{1}}{\lambda_{1}{ }^{n-2}} a_{n} \frac{b_{1} b_{2} \cdots b_{i-1} x_{1}}{\lambda_{1}{ }^{n-1}} \\
= & 0
\end{aligned}
$$




$$
\begin{aligned}
& x_{1}\left(\lambda_{1}-a_{1}-a=\frac{b_{1}}{\lambda_{1}}-a_{3}\right. \\
& =\frac{b_{2} x_{2}}{\lambda_{1}{ }^{2}}-\cdots-a_{n-1} \frac{b_{1} b_{2} \cdots b_{i-2}}{\lambda_{1}^{n-2}} \\
& \left.-a_{n} \frac{b_{1} b_{2} \cdots b_{i-1}}{\lambda_{1}^{n-1}}\right)=0
\end{aligned}
$$

then obtained

$$
x_{1}=0
$$

It is known that $\boldsymbol{x}_{\mathbf{1}}$ the nonzero vector corresponds to $\lambda_{1}$. From equation (12), if $\boldsymbol{x}_{\mathbf{1}}=0$, then the eigenvector corresponding to $\lambda_{1}$ is a zero vector. So much so that suppose $\boldsymbol{x}_{\mathbf{1}}=t$, it is obtained

$$
\begin{gathered}
x_{2}=\frac{b_{1} t}{\lambda_{1}} \\
x_{3}=\frac{b_{1} b_{2} t}{\lambda_{1}{ }^{2}} \\
x_{n-1}=\frac{b_{1} b_{2} \ldots b_{1-2} t}{\lambda_{1}{ }^{n-2}} \\
x_{n}=\frac{b_{1} b_{2} \ldots b_{1-1} t}{\lambda_{1}{ }^{n-1}}
\end{gathered}
$$

As a result, an eigenvector $\boldsymbol{x}_{\mathbf{1}}$ that corresponds to shape $\lambda_{1}$ is obtained

$$
\boldsymbol{x}_{\mathbf{1}}=\left[\begin{array}{c}
1 \\
\frac{b_{1}}{\lambda_{1}} \\
\vdots \\
\frac{b_{1} b_{2} b_{3} \ldots b_{1-2}}{\lambda_{1}{ }^{n-2}} \\
\frac{b_{1} b_{2} b_{3} \ldots b_{1-1}}{\lambda_{1}{ }^{n-1}}
\end{array}\right]
$$

with $t \in R-(0)$

Based on equation (13), it is found that the eigenvalue space $\boldsymbol{x}_{\mathbf{1}}$ has one dimension, so it can be concluded that the geometric multiplicity is equal to one and it is obtained that the elements of the eigenvector $x_{1}$ are positive numbers.

Theorem 2.2 If $\lambda_{1}$ is a single positive eigenvalue of a Leslie matrix and $\lambda_{1}$ is any real number eigenvalues or complex numbers of the Leslie matrix, then $\left|\lambda_{k}\right| \leq \lambda_{1}$

\section{Proof :}

Take any with $\lambda_{k}=r e^{i \theta} e^{i \theta}=\cos \cos \theta+\sin \sin \theta$ so that $\lambda_{k}=r \cos \cos \theta+i r \sin \sin \theta$.

It will be shown that $r<\lambda_{1}$

From equation (7) it is known $q(\lambda)=1$, so that it is obtained

$$
h\left(\lambda_{k}\right)=h\left(\lambda_{k}\right)=1
$$

$$
\begin{gathered}
\Leftrightarrow \frac{a_{1}}{r \cos \cos \theta+}+\frac{a_{2} b_{1} \sin \sin \theta}{(r \cos \cos \theta+i r \sin \sin \theta)^{2}} \\
\quad+\frac{a_{3} b_{1} b_{2}}{(r \cos \cos \theta+i r \sin \sin \theta)^{3}}+\cdots \\
+\frac{a_{3} b_{1} b_{2} \cdots b_{i-1}}{(r \cos \cos \theta+i r \sin \sin \theta)^{n}} \\
=\frac{a_{1}}{\lambda_{1}}+\frac{a_{2} b_{1}}{\lambda_{1}{ }^{2}}+\frac{a_{3} b_{1} b_{2}}{\lambda_{1}{ }^{3}+\cdots+\frac{a_{3} b_{1} b_{2} \cdots b_{i-1}}{\left(\lambda_{1}\right)^{n}}}=1 \\
\Leftrightarrow \frac{a_{1}}{\lambda_{1}}=\frac{a_{1}}{r \cos \cos \theta+i r \sin \sin \theta}
\end{gathered}
$$

by taking the real part of the two equations we get:

$$
\frac{a_{1}}{r \cos \cos \theta}=\frac{a_{1}}{\lambda_{1}}
$$

so that $r=\frac{\lambda_{1}}{\operatorname{coscos} \theta}$ and it can be concluded that

$$
r \leq \lambda_{1} \text { and }\left|\lambda_{k}\right| \leq \lambda_{1} \text {. }
$$

It is known that $r \leq \lambda_{1}$ it is evident that for anything $\lambda_{k}$ that is a real or complex number is valid $\left|\lambda_{k}\right| \leq \lambda_{1}$.

Definition 2.1 Given $\lambda_{1}, \lambda_{2}, \ldots, \lambda_{n}$ is the eigenvalue of matrix A of size $n \times n, \lambda_{1}$ is said to be the dominant eigenvalue of $\mathrm{A}$ if $\left|\lambda_{k}\right|>\left|\lambda_{i}\right|$ with $i=1,2, \cdots, n$.

Suppose that the Leslie A matrix can be diagonalized, then there is an eigenvalue, for example $\lambda_{1}, \lambda_{2}, \lambda_{2}, \ldots, \lambda_{n}$, and it has an eigenvector $x_{1}, x_{2}, x_{3}, \ldots, x_{n}$. Suppose that a matrix is formed

$$
P=\left[x_{1}\left|x_{2}\right| x_{3}|\ldots| x_{n}\right] \text {. }
$$

then there is $P^{-1}$ which is the inverse matrix of $P$. The diagonalization of the matrix $A$ takes the form:

$$
A=P\left[\begin{array}{cccc}
\lambda_{1} & 0 & \cdots & 0 \\
0 & \lambda_{2} & \cdots & 0 \\
\vdots & \vdots & \ddots & \vdots \\
0 & 0 & \cdots & \lambda_{n}
\end{array}\right] P^{-1}
$$

For $A^{p}$ with $p=1,2, \ldots, n$, the diagonalization equation becomes

$$
A^{p}=P\left[\begin{array}{cccc}
\lambda_{1}^{p} & 0 & \cdots & 0 \\
0 & \lambda_{2}^{p} & \cdots & 0 \\
\vdots & \vdots & \ddots & \vdots \\
0 & 0 & \cdots & \lambda_{n}^{p}
\end{array}\right] P^{-1}
$$

Suppose there is $\mathrm{n}(\mathrm{t})$ which is the vector of the initial distribution of a population, if multiplied by the diagonalization equation, then the equation becomes

$$
, A^{p} n(t)=P\left[\begin{array}{cccc}
\lambda_{1}^{p} & 0 & \cdots & 0 \\
0 & \lambda_{2}^{p} & \cdots & 0 \\
\vdots & \vdots & \ddots & \vdots \\
0 & 0 & \cdots & \lambda_{n}^{p}
\end{array}\right] P^{-1} n(t)
$$

For example, if the entry results in a multiplication 


$$
\begin{aligned}
& P^{-1} n(t)=\left[\begin{array}{c}
c_{1} \\
c_{2} \\
\vdots \\
c_{n}
\end{array}\right], \\
& \text { then } \\
& n(t+p)=\left[x_{1}\left|x_{2}\right| x_{3}|\ldots| x_{n}\right]\left[\begin{array}{cccc}
\lambda_{1}^{p} & 0 & \cdots & 0 \\
0 & \lambda_{2}^{p} & \cdots & 0 \\
\vdots & \vdots & \ddots & \vdots \\
0 & 0 & \cdots & \lambda_{n}^{p}
\end{array}\right]\left[\begin{array}{c}
c_{1} \\
c_{2} \\
\vdots \\
c_{n}
\end{array}\right] \\
& \Leftrightarrow n(t+p)=\left[x_{1}\left|x_{2}\right| x_{3}|\ldots| x_{n}\right]\left[\begin{array}{c}
\lambda_{1}^{p} c_{1} \\
\lambda_{2}^{p} c_{2} \\
\vdots \\
\lambda_{n}^{p} c_{n}
\end{array}\right] \\
& \Leftrightarrow n(t+p)=x_{1} \lambda_{1}{ }^{P} c_{1}+x_{2} \lambda_{2}{ }^{P} c_{2}+\cdots+x_{n} \lambda_{n}{ }^{P} c_{n}
\end{aligned}
$$

It will be shown that if $\lambda_{1}$ it is the dominant eigenvalue it will affect population growth. The two sides are divided by $\lambda_{1}{ }^{P}$, then the equation becomes

$$
\frac{1}{\lambda_{1}{ }^{P}} n(t+p)=P\left[\begin{array}{cccc}
1 & 0 & \cdots & 0 \\
0 & \left(\frac{\lambda_{2}}{\lambda_{1}}\right)^{p} & \cdots & 0 \\
\vdots & \vdots & & \ddots \\
0 & 0 & \cdots & \left(\frac{\lambda_{n}}{\lambda_{1}}\right)^{p}
\end{array}\right] P^{-1} n(t)
$$

It is known that $\lambda_{1}$ the dominant eigenvalues of the Leslie matrix, then $\left(\frac{\lambda_{n}}{\lambda_{1}}\right)$, for $n=1,2, \ldots, n$, and are obtained $\left(\frac{\lambda_{n}}{\lambda_{1}}\right)^{p} \rightarrow 0$ when $p \rightarrow \infty$.

A limit is formed

$$
\begin{aligned}
& \lim _{p \rightarrow \infty} \frac{1}{\lambda_{1}{ }^{P}} n(t+p) \\
= & P\left[\begin{array}{cccc}
1 & 0 & \cdots & 0 \\
0 & 0 & \cdots & 0 \\
\vdots & \vdots & \ddots & \vdots \\
0 & 0 & \cdots & 0
\end{array}\right] P^{-1} n(t)
\end{aligned}
$$

Given that $P^{-1}$ is a matrix with the order $n x n$, and $n(t)$ the vector of the initial distribution with the order $n \times 1$. If $P^{-1}$ and $n(t)$ are multiplied according to the multiplication properties of the matrix, then the matrix result will be ordered $n x 1$. Both the $P^{-1}$ and $n(t)$ matrices have entries which are constants. Substituting equation (14) to equation (15), is obtained

$$
\begin{gathered}
\Leftrightarrow \lim _{p \rightarrow \infty} \frac{1}{\lambda_{1}{ }^{P}} n(t+p)=P\left[\begin{array}{cccc}
1 & 0 & \cdots & 0 \\
0 & 0 & \cdots & 0 \\
\vdots & \vdots & \ddots & \vdots \\
0 & 0 & \cdots & 0
\end{array}\right]\left[\begin{array}{c}
c_{1} \\
c_{2} \\
\vdots \\
c_{n}
\end{array}\right] \\
\Leftrightarrow \lim _{p \rightarrow \infty} \frac{1}{\lambda_{1}{ }^{P}} n(t+p) \\
\quad=\left[x_{1}\left|x_{2}\right| x_{3}|\ldots| x_{n}\right]\left[\begin{array}{cccc}
1 & 0 & \cdots & 0 \\
0 & 0 & \cdots & 0 \\
\vdots & \vdots & \ddots & \vdots \\
0 & 0 & \cdots & 0
\end{array}\right]\left[\begin{array}{c}
c_{1} \\
c_{2} \\
\vdots \\
c_{n}
\end{array}\right]
\end{gathered}
$$

$$
\begin{gathered}
\Leftrightarrow \lim _{p \rightarrow \infty} \frac{1}{\lambda_{1}{ }^{P}} n(t+p)=\left[x_{1}\left|x_{2}\right| x_{3}|\ldots| x_{n}\right]\left[\begin{array}{c}
c_{1} \\
0 \\
\vdots \\
0
\end{array}\right] \\
\Leftrightarrow \lim _{p \rightarrow \infty} \frac{1}{\lambda_{1}{ }^{P}} n(t+p)=c_{1} x_{1}+0 x_{2}+0 x_{3} \ldots+0 x_{n} \\
\Leftrightarrow \lim _{p \rightarrow \infty} \frac{1}{\lambda_{1}{ }^{P}} n(t+p)=c_{1} x_{1}
\end{gathered}
$$

From equation (16) an approximation is obtained

$$
\begin{gathered}
n(t+p)=c_{1} \lambda_{1}{ }^{p} x_{1} \\
\Leftrightarrow n(t+p)=c_{1} \lambda_{1}{ }^{p-1} x_{1}
\end{gathered}
$$

such that $n(t+p)$

$$
\begin{gathered}
=\lambda_{1}{ }^{P} c_{1} x_{1} \Leftrightarrow n(t+p)=c_{1}{ }^{1} \lambda_{1}{ }^{p-1} c_{1} x_{1} \\
\text { obtained } n(t+p)=\lambda_{1} n(t+(p-1))
\end{gathered}
$$

From equation (17), it is found that, if for any value $p$ that determines the next year in the population, and it is known that $\lambda_{1}=1$ the dominant eigenvalue of the Leslie matrix, it can be concluded that the next age distribution vector will always be the same as the previous age vector. So as to result:

1. If $\lambda_{1}<1$ is known, the population of all age classes tends to decline.

2. If $\lambda_{1}=1$ is known, the population in all age classes tends to remain.

3. If $\lambda_{1}>1$ is known, the population of all age classes tends to increase.

\section{Research Result}

In Table 2. below, data obtained from the BPS of Medan city is provided, namely the number of female residents in 2010 and 2015 in the city of Medan, grouped by age.

Data obtained from BPS are data on female population by age, data on the birth of girls according to maternal age at delivery, and data on female population mortality. The data is arranged in the following frequency distribution table. 
Table 2. Female Population in Medan City 2010 and 2015

\begin{tabular}{|c|c|c|}
\hline Age Class & $\begin{array}{c}\text { Number of Women in } \\
2010\end{array}$ & Number of Women in 2015 \\
\hline $0-4$ & 92,857 & 99,065 \\
\hline $5-9$ & 93,532 & 95,441 \\
\hline $10-14$ & 91,828 & 89,405 \\
\hline $15-19$ & 107,423 & 109,850 \\
\hline $20-24$ & 123,092 & 128,830 \\
\hline $25-29$ & 103,459 & 100,090 \\
\hline $30-34$ & 87,265 & 90,398 \\
\hline $35-39$ & 80,759 & 84,551 \\
\hline $40-44$ & 71,727 & 75,953 \\
\hline $45-49$ & 59,997 & 65,817 \\
\hline $50-54$ & 49,244 & 56,676 \\
\hline $55-59$ & 34,282 & 45,175 \\
\hline $60-64$ & 22,555 & 31,355 \\
\hline $65-69$ & 17,556 & 19,903 \\
\hline $70-74$ & 12,384 & 13,714 \\
\hline $75+$ & 12,688 & 12,364 \\
\hline Total & $1,060,648$ & $1,118,587$ \\
\hline & & \\
\hline
\end{tabular}

From Table 3, the initial population of women in Medan is $1,060,648$ people, the number of female births $\left(A_{i}\right)$ is 270,612 and the number of female population deaths $(\mathrm{Bi})$ is 6,597 .

Furthermore, the Leslie matrix model is formed, therefore the element of female fertility level is needed, for example $\left(a_{i}\right)$ and the element of female survival rate $\left(b_{i}\right)$. The formula for finding ai and bi is as follows.

$$
a_{i}=\frac{A_{i}}{x_{i}^{0}} \text { and } b_{i}=1-\frac{B_{i}}{x_{i}^{0}}
$$

by:

$A_{i}=$ Number of births in age class to $-i$

$B_{i}=$ Number of female deaths in age class to $-i$

$X_{i}^{0}=$ The number of initial population of women in age class to $-i$
Table 3. Female Population in Medan, 2010-2015

\begin{tabular}{|c|c|c|c|c|}
\hline $\begin{array}{c}\text { Age } \\
\text { Class }\end{array}$ & $\begin{array}{c}\text { Age } \\
\text { Interval }\end{array}$ & $\begin{array}{c}\text { Number of } \\
\text { Women in } \\
2010\left(x_{i}^{0}\right)\end{array}$ & $\begin{array}{c}\text { Birth } \\
\left(A_{i}\right)\end{array}$ & $\begin{array}{c}\text { Dead } \\
\left(B_{i}\right)\end{array}$ \\
\hline 1 & $0-4$ & 92,857 & 0 & 527 \\
\hline 2 & $5-9$ & 93,532 & 0 & 483 \\
\hline 3 & $10-14$ & 91,828 & 0 & 638 \\
\hline 4 & $15-19$ & 107,423 & 21,188 & 712 \\
\hline 5 & $20-24$ & 123,092 & 62,544 & 487 \\
\hline 6 & $25-29$ & 103,459 & 80,023 & 512 \\
\hline 7 & $30-34$ & 87,265 & 48,995 & 338 \\
\hline 8 & $35-39$ & 80,759 & 40,171 & 498 \\
\hline 9 & $40-44$ & 71,727 & 17,691 & 446 \\
\hline 10 & $45-49$ & 59,997 & 0 & 310 \\
\hline 11 & $50-54$ & 49,244 & 0 & 272 \\
\hline 12 & $55-59$ & 34,282 & 0 & 304 \\
\hline 13 & $60-64$ & 22,555 & 0 & 385 \\
\hline 14 & $65-69$ & 17,556 & 0 & 367 \\
\hline 15 & $70-74$ & 12,384 & 0 & 171 \\
\hline 16 & $75+$ & 12,688 & 0 & 147 \\
\hline & total & $1,060,648$ & 270,612 & 6,597 \\
\hline
\end{tabular}

Table 4. Levels of Fertility and Survival Women in Medan in 2010 2015

\begin{tabular}{|c|c|c|}
\hline Age Class & $\begin{array}{c}\text { Fertility Rate } \\
\left(a_{i}\right)\end{array}$ & $\begin{array}{c}\text { Level of Resistance } \\
\left(b_{i}\right)\end{array}$ \\
\hline 1 & 0 & 0.9944 \\
\hline 2 & 0 & 0.9949 \\
\hline 3 & 0 & 0.9931 \\
\hline 4 & 0.196 & 0.9934 \\
\hline 5 & 0.508 & 0.9961 \\
\hline 6 & 0.773 & 0.9951 \\
\hline 7 & 0.561 & 0.9962 \\
\hline 8 & 0.497 & 0.9939 \\
\hline 9 & 0.246 & 0.9938 \\
\hline 10 & 0 & 0.9949 \\
\hline 11 & 0 & 0.9945 \\
\hline 12 & 0 & 0.9912 \\
\hline 13 & 0 & 0.983 \\
\hline 14 & 0 & 0.9791 \\
\hline 15 & 0 & 0.9862 \\
\hline 16 & 0 & 0.9885 \\
\hline
\end{tabular}

From Table 4, above, the Leslie matrix model is obtained. The resulting Leslie matrix is a matrix with the order of $16 \times 16$ whose elements consist of the female fertility rate $\left(a_{i}\right)$ in the first row and the female survival rate $\left(b_{i}\right)$ in the diagonal row of the female population. 


$$
L=\left[\begin{array}{cccccccccccccccc}
0 & 0 & 0 & 0.196 & 0.508 & 0.773 & 0.561 & 0.497 & 0.246 & 0 & 0 & 0 & 0 & 0 & 0 & 0 \\
09944 & 0 & 0 & 0 & 0 & 0 & 0 & 0 & 0 & 0 & 0 & 0 & 0 & 0 & 0 & 0 \\
0 & 0.9949 & 0 & 0 & 0 & 0 & 0 & 0 & 0 & 0 & 0 & 0 & 0 & 0 & 0 & 0 \\
0 & 0 & 0.9931 & 0 & 0 & 0 & 0 & 0 & 0 & 0 & 0 & 0 & 0 & 0 & 0 & 0 \\
0 & 0 & 0 & 0.9934 & 0 & 0 & 0 & 0 & 0 & 0 & 0 & 0 & 0 & 0 & 0 & 0 \\
0 & 0 & 0 & 0 & 0.9961 & 0 & 0 & 0 & 0 & 0 & 0 & 0 & 0 & 0 & 0 & 0 \\
0 & 0 & 0 & 0 & 0 & 0.9951 & 0 & 0 & 0 & 0 & 0 & 0 & 0 & 0 & 0 & 0 \\
0 & 0 & 0 & 0 & 0 & 0 & 0.9962 & 0 & 0 & 0 & 0 & 0 & 0 & 0 & 0 & 0 \\
0 & 0 & 0 & 0 & 0 & 0 & 0 & 0.9939 & 0 & 0 & 0 & 0 & 0 & 0 & 0 & 0 \\
0 & 0 & 0 & 0 & 0 & 0 & 0 & 0 & 0.9938 & 0 & 0 & 0 & 0 & 0 & 0 & 0 \\
0 & 0 & 0 & 0 & 0 & 0 & 0 & 0 & 0 & 0.9949 & 0 & 0 & 0 & 0 & 0 & 0 \\
0 & 0 & 0 & 0 & 0 & 0 & 0 & 0 & 0 & 0 & 0.9945 & 0 & 0 & 0 & 0 & 0 \\
0 & 0 & 0 & 0 & 0 & 0 & 0 & 0 & 0 & 0 & 0 & 0.9912 & 0 & 0 & 0 & 0 \\
0 & 0 & 0 & 0 & 0 & 0 & 0 & 0 & 0 & 0 & 0 & 0 & 0.983 & 0 & 0 & 0 \\
0 & 0 & 0 & 0 & 0 & 0 & 0 & 0 & 0 & 0 & 0 & 0 & 0 & 0.9791 & 0 & 0 \\
0 & 0 & 0 & 0 & 0 & 0 & 0 & 0 & 0 & 0 & 0 & 0 & 0 & 0 & 0.98620
\end{array}\right]
$$

then the predicted number of female population in 2025 is:

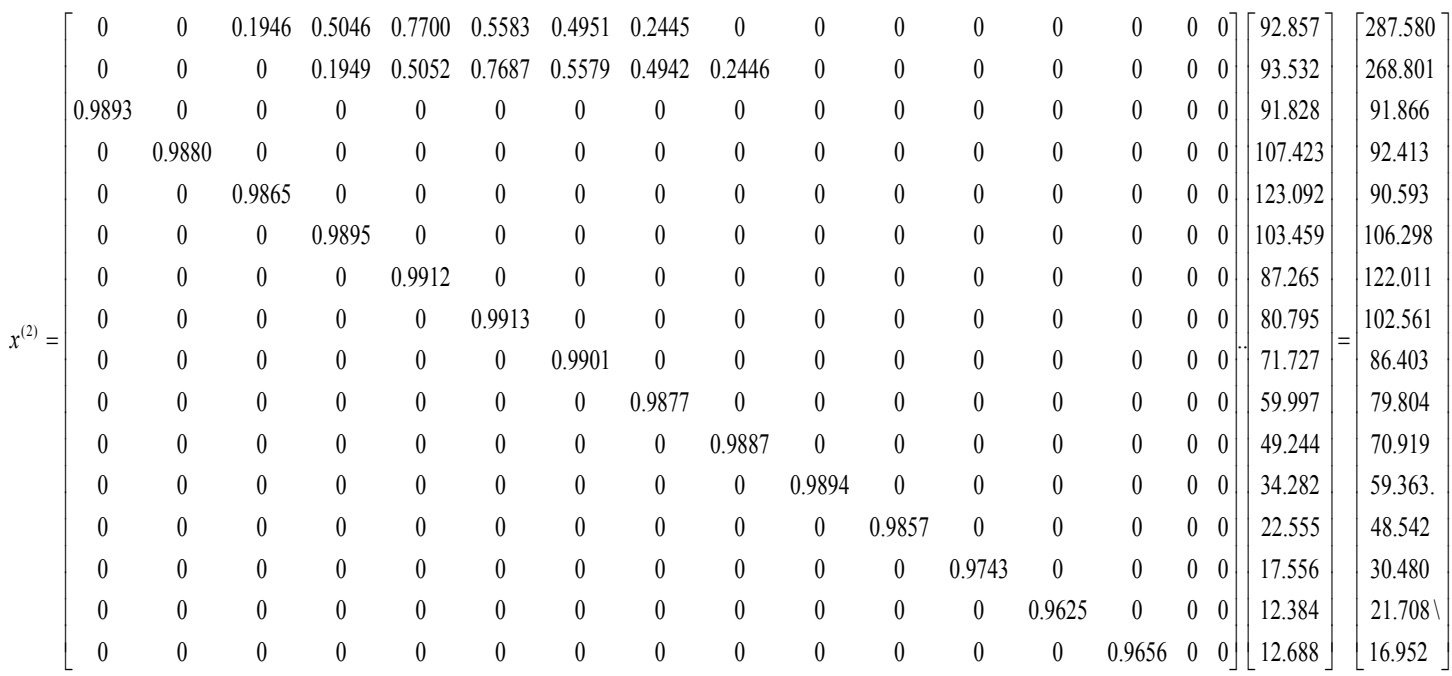

$$
\begin{aligned}
& x^{(2)}=287.580+268.801+91.866+92.413+90.593+106.298+122.011+102.561+ \\
& 86.403+79.804+70.919+59.363+48.542+30.480+21.708+16.952=1.576 .294
\end{aligned}
$$

To find out the prediction of the growth rate of the female population in the city of Medan, a search for eigenvalues was carried out. Because the $\mathrm{L}$ matrix is quite large, the authors looked for the eigenvalues of the Leslie matrix with the help of matlab software and obtained:

$\lambda_{1}=1.3673, \lambda_{2}=0.4724+0.1704 i, \lambda_{3} 0.7424-$ $0.1704 i, \quad \lambda_{4}=-0.3623+0.9047 i, \quad \lambda_{5}=-0.3623-$ $0.9047 \mathrm{i}, \lambda_{6}=-0.2055+0.6619 \mathrm{i}, \lambda_{7}=-0.2055-$ $0.6619 \mathrm{i}, \lambda_{8}=-0.5883+0.0829 \mathrm{i}, \lambda_{9}=-0.5883-$ $0.0829 \mathrm{i}, \lambda_{10}=0, \lambda_{11}=0, \lambda_{12}=0, \lambda_{13}=0, \lambda_{14}=0$, $\lambda_{15}=0, \lambda_{16}=0$ and dominan eigenvalue is $\lambda_{1}=$ 1.3673. So it can be concluded that the female population growth in the city of Medan will ten to increase.

\section{Conclusions}

Based on the results and discussion, the following conclusions were obtained:

a). In the prediction analysis the population size obtained $x^{(2)}=1,576,294$. So it can be seen that the number of female population in 2025 has increased.

b). The results of the application of the Leslie matrix obtained the eigen value or $\lambda_{1}>1$. This indicates that the number of female population in Medan City in 2025 will increase. 


\section{REFERENCES}

[1] BPS - Statistics of Medan City, Medan City in Figures 2010, BPS Medan City, Medan, 2010.

[2] BPS - Statistics of Medan City, Medan City in Figures 2015, BPS Medan City, Medan, 2015.

[3] Dewi Anggreini, The Female Population Growth Projection Regency by Leslie Matix Model on the Birth, Biology, Medicine \& Natural Product Chemistry, Vol. 6, No.2, pp. $37-45,2017$

[4] Dewi Anggreini, Leslie Matrix Model with Harvesting Strategies in the Youngest Age Group on Female Sheep Fertility and Life Expectancy, Fourier Journal, Vol. 7, No.1, pp. 23-34, 2018.

[5] Howard Anton and Chris Rorres, Elementary Linear Algebra, John Wiley \& Sons, Inc., 2014.
[6] Leslie Matrices, Online available from https://www.math.u h.edu/ klaus/Leslie\%20Matrices_final_corrected.pdf

[7] Michael Monagan, Using Leslie matrices as the application of eigenvalues and eigenvectors in a first course in Linear Algebra, Online available from http://www.cecm.sfu.ca/ m monagan/papers/Leslie3.pdf

[8] Yudha Pratama, Bayu Prihandoko and Nilamsari Kusumastuti, Application of the Leslie Matrix to Predict the Number and Growth Rate of a Population, Scientific Bulletin Math.Stat.and Its Applications (Bimaster), Vol. 02, No.3, pp. 163-172, 2013.

[9] Wahidah Sanusi, Sukarna and Nur Ridiawati, The Leslie Matrix and Its Application in Predicting Population Growth Rate and Amount in Makassar City, Journal of Mathematics, Computations, and Statistics, Vol.1, No.2, pp. 142- 154, 2018. 